\title{
The Impact of landscape composition for urban heat island intensity in Addis Ababa City using Landsat data (1986-2016)
}

\author{
DMSLB Dissanayake $^{\mathrm{a}, \mathrm{b}, *}$,Takehiro Morimoto ${ }^{\mathrm{c}}$, Yuji Murayama ${ }^{\mathrm{c}}$, Manjula Ranagalage ${ }^{\mathrm{a}, \mathrm{b}}$ \\ ${ }^{a}$ Graduate School of Life and Environmental Sciences, University of Tsukuba, 1-1-1, Tennodai, Tsukuba, Ibaraki 305-8572, Japan; \\ dissanayakedmslb@gmail.com,manjularanagalage@gmail.com \\ ${ }^{b}$ Department of Environmental Management, Faculty of Social Sciences and Humanities, Rajarata University of Sri Lanka, \\ Mihintale 50300, Sri Lanka \\ ${ }^{c}$ Faculty of Life and Environmental Sciences, University of Tsukuba, 1-1-1, Tennodai, Tsukuba, Ibaraki 305-8572, Japan; \\ tmrmt@geoenv.tsukuba.ac.jp, mura@geoenv.tsukuba.ac.jp
}

* DMSLB Dissanayake

Keywords: Surface urban heat island intensity, Urban rural gradient analysis, Land surface temperature, Addis Ababa

\begin{abstract}
:
Exploring changes in land use and land cover (LULC) in the city area and its surrounding is important to understand the variation of surface urban heat island (SUHI) and surface urban heat island intensity (SUHII). The SUHII can be calculated based on the local climate zone by using land use and land cover compossition of the city and based on the urban rural zone. The objective of this research is to examine the spatiotemporal changes of LULC and the impact of its composition for the formation of SUHI in Addis Ababa City, Ethiopia based on the urban rural zones.

The mean center of the central business district of the Addis Ababa City was considered as the central point of the study area. We represented the $30 \mathrm{~km} \times 30 \mathrm{~km}$ geographical area as a study area with a $15 \mathrm{~km}$ radius from the central point. As data sources, multi-temporal satellite data provided by the United States Geological Survey (USGS) were used in respect to the years of 1986, 2001, and 2016. In the methodology, we first completed the classification of LULC by using pixeloriented method for the three years and the validation of the classification has been made. For the classification five LULC classes were identified such as forest, impervious surface, grass land, bare land and crop land. Afterward, land surface temperature (LST) has been computed for three years respectively. Finally, urban rural gradient zones (URGZs) have been generated as a set of polygons with $210 \mathrm{~m}$ distance in each zone from the central point of the study area. In order to evaluate the SUHII along the URGZs in respect to the LULC, the following analyses were accomplished: (i) the relationship between mean LST and composition of the LULC was computed, (ii) the SUHII was calculated based on the LST variation of main LULC categories and the temperature difference between URGZs, (iii) multi-temporal and multidirectional SUHII was computed, and (iv) linear regression analyses were used to assess the correlations of the mean LST with composition of LULC.

The results of the analyses show that (i) distribution pattern of SUHII has changed over the study period as results of changes in LULC, and (ii) mean LST gradually declines from city centre to outside of the city, then it can be seen increasing trends due to the effect of bare lands in rural area. This pattern can be seen over the three years as the result of multi-directional approach. The methodology presented will be able to apply other cities which are showing similar growth pattern by making necessary calibration, and our finding can be used as a proxy indicator to introduce appropriate landscape and town planning in a sustainable viewpoint in Addis Ababa City.
\end{abstract}

